\title{
Polarized single photon emission and photon bunching from an InGaN quantum dot on a GaN micropyramid
}

\author{
Tomas Jemsson, Houssaine Machhadani, Per-Olof Holtz and Fredrik K Karlsson
}

\author{
Linköping University Post Print
}

\section{Tweet}

N.B.: When citing this work, cite the original article.

Original Publication:

Tomas Jemsson, Houssaine Machhadani, Per-Olof Holtz and Fredrik K Karlsson, Polarized single photon emission and photon bunching from an InGaN quantum dot on a $\mathrm{GaN}$ micropyramid, 2015, Nanotechnology, (26), 6, 065702.

http://dx.doi.org/10.1088/0957-4484/26/6/065702

Copyright: IOP Publishing: Hybrid Open Access http://www.iop.org/

Postprint available at: Linköping University Electronic Press

http://urn.kb.se/resolve?urn=urn:nbn:se:liu:diva-113347 


\title{
Polarized single photon emission and photon bunching from an InGaN quantum dot on a GaN micropyramid
}

\author{
Tomas Jemsson,* Houssaine Machhadani, Per-Olof Holtz, and K. Fredrik Karlsson \\ Department of Physics, Chemistry, and Biology (IFM), \\ Semiconductor Materials, Linköping University, S-58183 Linköping, Sweden
}

(Dated: January 11, 2015)

\begin{abstract}
We report on excitonic single photon emission and biexcitonic photon bunching from an InGaN quantum dot formed on the apex of a hexagonal GaN micropyramid. An approach to suppress uncorrelated emission from the pyramid base is proposed, a metal film is demonstrated to effectively screen background emission and thereby significantly enhance the signal-to-background ratio of the quantum dot emission. As a result, the second order coherence function at zero time delay $g^{(2)}(0)$ is significantly reduced (to $g^{(2)}(0)=0.24$, raw value) for the excitonic autocorrelation at a temperature of $12 \mathrm{~K}$ under continuous wave excitation, and a dominating single photon emission is demonstrated to survive up to $50 \mathrm{~K}$. The deterioration of the $g^{(2)}(0)$-value at elevated temperatures is well understood as the combined effect of reduced signal-to-background ratio and limited time resolution of the setup. This result underlines the great potential of site controlled pyramidal dots as sources of fast polarized single photons.
\end{abstract}

\section{INTRODUCTION}

Generation of non-classical light is both of fundamental interest and a common condition for quantum information applications (QIA). Semiconductor quantum dots (QDs) are feasible photon emitters for QIA due to their atomic-like energy structure and their possibility to be integrated with other semiconductor devices on the same chip. Site-controlled QDs operating close to room temperature are demanded for widespread applications, and linearly polarized emitters are a prerequisite for certain QIA $[1,2]$.

III-nitride QDs have attractive properties in terms of deep confinement potentials needed for high temperature operation, whilst the small split-off energy of the nitrides causes a high degree of linear polarization already for weakly asymmetric quantum dots [3]. The demonstration of single photon emission at room temperature was recently reported for a GaN QD [4]. Also single photon biexcitonic emission from an InGaN QD in a nanowire at $200 \mathrm{~K}[5]$ and an electrically driven InGaN QD in a nanowire exhibiting single photon excitonic emission at $150 \mathrm{~K}[6]$ have been reported.

A promising approach for fabrication of site-controlled nitride-based QDs is to deposit a thin layer of InGaN on top of hexagonal GaN micropyramids [7]. QDs formed on the apex of the pyramids grown with this approach have been shown to exhibit single and sharp InGaN related emission lines with a high degree of linear polarization. A simple elongation of the pyramid base gives control of the polarization direction $[8,9]$. The photon statistics of these pyramidal QDs have recently been shown to exhibit anti-bunching, but an spectrally overlapping background emission of uncorrelated photons will vanish high-performance single photon characteristics of the

\footnotetext{
* tomje@ifm.liu.se
}

emission [10]. Cathodoluminescence measurements have revealed that the broad background emission mainly originates from the bottom edges near the pyramid base [9].

A remedy to this problem of background emission is proposed by adding a post growth process to cover the lower parts of the pyramid sides as well as the area between the pyramids with a metal film. This reduces the background emission and largely improves the relative QD signal, $\rho=\frac{S}{S+B}$, where $S(B)$ is the measured signal (uncorrelated background) intensity. As a result, significantly improved single photon characteristics were demonstrated.

\section{EXPERIMENTAL DETAILS}

The structure used in this investigation was grown by a horizontal low pressure hot-wall metal-organic chemical vapor deposition system. Selective area growth of GaN pyramids was performed on a lithographically patterned SiN masked $\mathrm{GaN} / \mathrm{AlN} / \mathrm{SiC}$ template [8]. The pyramids were covered by a thin layer of InGaN, from which nanoscopic 3D islands in a Stranski-Krastanov-like growth mode will be formed on the small (0001) top surface of slightly truncated pyramids. Finally, a capping layer of GaN was grown [7]. The resulting pyramids have a base diameter of $3 \mu \mathrm{m}$ and are ordered in square $21 \times 21$ arrays with $5.5 \mu \mathrm{m}$ pitch.

The process to cover the lower parts of the hexagonal GaN pyramids with metal was performed in four steps. First a layer of $\mathrm{Al}$ was sputtered on the pyramids with a nominal thickness of $200 \mathrm{~nm}$, referring to the measured thickness on a planar sample, followed by spin coating of Hydrogen Silsesquioxane (HSQ) resist on top of the $\mathrm{Al}$ layer. Thermal annealing of HSQ at $470{ }^{\circ} \mathrm{C}$ under a protective $\mathrm{N}_{2}$ gas flow during one hour transferred the HSQ resist into $\mathrm{SiO}_{2}$. The thickness of HSQ was chosen not to completely cover the pyramids with $\mathrm{SiO}_{2}$. Finally, the sample was immersed in an Al-etch solution 
for 6 minutes, removing the $\mathrm{Al}$ layer from the top part of the pyramids not covered with $\mathrm{SiO}_{2}$. In this simple process, which does not rely on conventional photo- or e-beam lithography and crucial alignments, all pyramids were completed with the metal mask simultaneously. The insert of figure $1 \mathrm{~b}$ shows a scanning electron microscopy (SEM) image of a pyramid after these processing steps.

A $355 \mathrm{~nm}$ continuous wave diode pumped solid state laser was used for the measurements. The excitation laser was led through a refractive microscope objective (NA = 0.42 ) and focused down to a spot size of about $1 \mu \mathrm{m}$ on the sample, allowing excitation of one single pyramid. The sample was mounted on the cold finger of a continuous flow helium cryostat, with the ability to reach temperatures down to $4 \mathrm{~K}$. The laser power used was in the range of 10 to $160 \mu \mathrm{W}$, as measured before the objective. The photons emitted from the sample were collected by the same objective and guided through a monochromator with the focal length of $550 \mathrm{~mm}$, allowing a spectral resolution of $0.28 \mathrm{meV}$. Micro-photoluminescence $(\mu \mathrm{PL})$ spectra were recorded, either by a charge coupled device (CCD) or by a photo multiplier tube (PMT) [11] operating in the photon counting mode. Time correlated single photon spectroscopy (TCSPS) measurements were performed in the same setup converted to a HanburyBrown and Twiss interferometer (HBT) by a beam splitter in the signal path, guiding half of the signal through another monochromator to a second PMT [12]. Both monochromators allowed filtering of the $\mu \mathrm{PL}$-signal, before it reached the PMTs, with a band pass of $0.11 \mathrm{~nm}$ for the relevant wavelengths. The time differences between the arrival of subsequent photons onto the PMTs were recorded by a TCSPC module with the overall instrument time constant of $\tau_{i}=0.12 \mathrm{~ns}$. The statistical occurrence of measured time differences were compiled into histograms with the bin width set to 128 ps for all experiments except the one performed at $80 \mathrm{~K}$, for which a larger bin width, $256 \mathrm{ps}$, was used. All TCSPS measurements were performed after the metallization process. It should be noted that we here have a factor 2.5 higher spectral resolution and six times faster detectors than in our previous work [10].

\section{RESULTS AND DISCUSSION}

Before the metallization process, the PL-signal from the investigated $\mathrm{QD}$ was spectrally overlapping with a strong background emission corresponding to $\rho \lesssim 0.57$. The intensity of this background emission was reduced by a factor of 20 after the metallization, causing an increase of relative signal intensity up to $\rho \approx 0.92$ (see figure 1a). The main reasons for this are that the metal film screens the excitation of the bottom pyramid edges and blocks any remaining background emission from this region. Despite the fact that the absolute intensity of the QD was reduced by a factor 2 after the metallization, possibly due to incomplete etching with remnants of the $\mathrm{Al}$ layer, the QD signal remains relatively strong with $\sim 40000$ registered counts per second for the dominating emission line. The predominant peak $\mathrm{X}$ at low excitation powers is attributed to the single exciton. For higher powers, a second peak XX, downshifted by $4.5 \mathrm{meV}$ vs. X, interpreted as the biexciton, gains intensity and becomes comparable to that of the exciton. A power dependence of X and XX (figure 1b) shows the typical linear and superlinear behaviors expected for the exciton and biexciton, respectively. However, it should be noted that a superlinear power dependence alone cannot be used to strictly link XX to the biexciton, since also trions may exhibit a nearly quadratic power dependence at low temperatures [13]. Both the X and XX peaks do exhibit a high degree of linear polarization (see inserts of figure 2a) along the same polarization direction, indicating that both emission lines originate from the same QD [14]. Besides the $\mathrm{X}$ and $\mathrm{XX}$ peaks, a third high-energy peak is observed at $\sim 3.246 \mathrm{eV}$. This peak is concluded to originate from another QD as its emission is uncorrelated with $\mathrm{X}$. There is also a weak low energy shoulder on XX but characterized by a polarization direction different from that of $\mathrm{X}$ and $\mathrm{XX}$ and, therefore, concluded to originate from another QD. Under optimized growth conditions, most pyramids accommodate only one QD each [8] but this particular pyramid reveals spectral features of more than one QD. A small pyramid-to-pyramid variation of the top surface may be the reason for this, as truncated pyramids with intentionally larger top surface are known to accommodate more QDs [9].

The $\mu \mathrm{PL}$ spectrum shown in figure $2 \mathrm{a}$ is acquired with a PMT in the photon counting mode, at the same experimental conditions as for the autocorrelation measurements of $\mathrm{X}$ shown in figure $2 \mathrm{~b}$. The experiment reveals a small value of the second order coherence function $g_{\exp }^{(2)}(0)=0.24$, which implies that the emission is dominated by single photons from the investigated QD.

Autocorrelation histograms of X measured at $50 \mathrm{~K}$ and $80 \mathrm{~K}$ (Figs. 3a and b) demonstrate higher $g_{\exp }^{(2)}(0)$ values, but single photon emission still dominates at $50 \mathrm{~K}$ with $g_{e x p}^{(2)}(0)=0.45$, and clear anti-bunching is observed at 80 $\mathrm{K}$ with $g_{\exp }^{(2)}(0)=0.63$. The background level in the spectra measured at elevated temperatures (figures $3 \mathrm{c}$ and $\mathrm{d}$ ) are similar to the low-temperature measurement at 12 $\mathrm{K}$ (figure 2a) despite the fact that the excitation power is higher. However, the peak intensity of the QD signal drops significantly as the temperature is elevated while the emission lines broaden.

It was earlier argued that the exciton and biexciton emission lines from the same QD do exhibit almost identical polarization properties [14]. We have here monitored a polarization dependence (inserts of figure 2a) which is in consistence with the nearly identical polarization behavior for the exciton and biexciton. Furthermore, the XX-X cross-correlation histogram (figure 2c) which exhibits the typical asymmetric bunching pattern, that proves biexciton emission, is a strong and direct experimental support for the arguments given in Ref. 14 . 


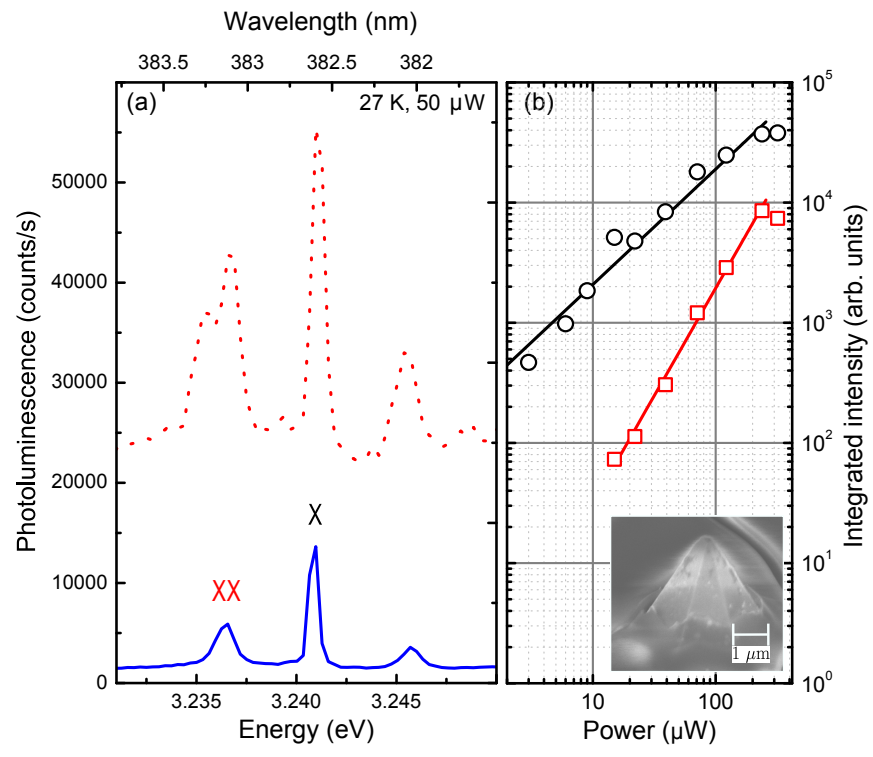

FIG. 1. (Color online) a) Two $\mu \mathrm{PL}$ spectra of the QD recorded with a CCD before (dotted red line) and after (solid blue line) the metallization process. The exciton and biexciton emissions are labeled X and XX in the figure. b) Power dependence of the integrated intensity of $\mathrm{X}$ (black circles) and $\mathrm{XX}$ (red squares) performed at $25 \mathrm{~K}$. The lines represent the best fit to the low power data with the exponents 1.0 and 1.8 , respectively. The insert shows a SEM picture of a hexagonal GaN pyramid with the lower part of its sides covered by Al.

Further analysis of the experimental data requires a mathematical formulation of the autocorrelation second order coherence function $g^{2}(\tau)$, as provided in Ref. 15, with correction for the superimposed uncorrelated background emission,

$$
g^{(2)}(\tau)=1-\rho^{2} e^{-|\tau| / \tau_{c}},
$$

where $\tau$ is the time difference between subsequent photons and $\tau_{c}$ is the characteristic anti-bunching time constant given by

$$
1 / \tau_{c}=1 / \tau_{x}+W,
$$

with the exciton lifetime $\tau_{x}$ and the effective pump rate $W[16,17]$. Convoluting 1 with the temporal impulse response function of the TCSPS apparatus, involving the instrument time constant $\tau_{i}$, the measured second order autocorrelation function $g_{m}^{(2)}(\tau)$ can be calculated given the three parameters $\rho, \tau_{x}$ and $\tau_{i}$ by,

$$
g_{m}^{(2)}(\tau)=\frac{1}{2 \tau_{i}} \int_{-\infty}^{+\infty} g^{(2)}(\tau) e^{-|\tau-t| / \tau_{i}} d t
$$

Finally, for non-zero histogram bin width $\tau_{B W}$, the measured value $g_{m}^{(2)}[0]$ at zero time difference is obtained by integrating $g_{m}^{(2)}(\tau)$ symmetrically around zero [10],

$$
g_{m}^{(2)}[0]=\frac{1}{\tau_{B W}} \int_{-\tau_{B W} / 2}^{+\tau_{B W} / 2} g_{m}^{(2)}(\tau) d \tau .
$$
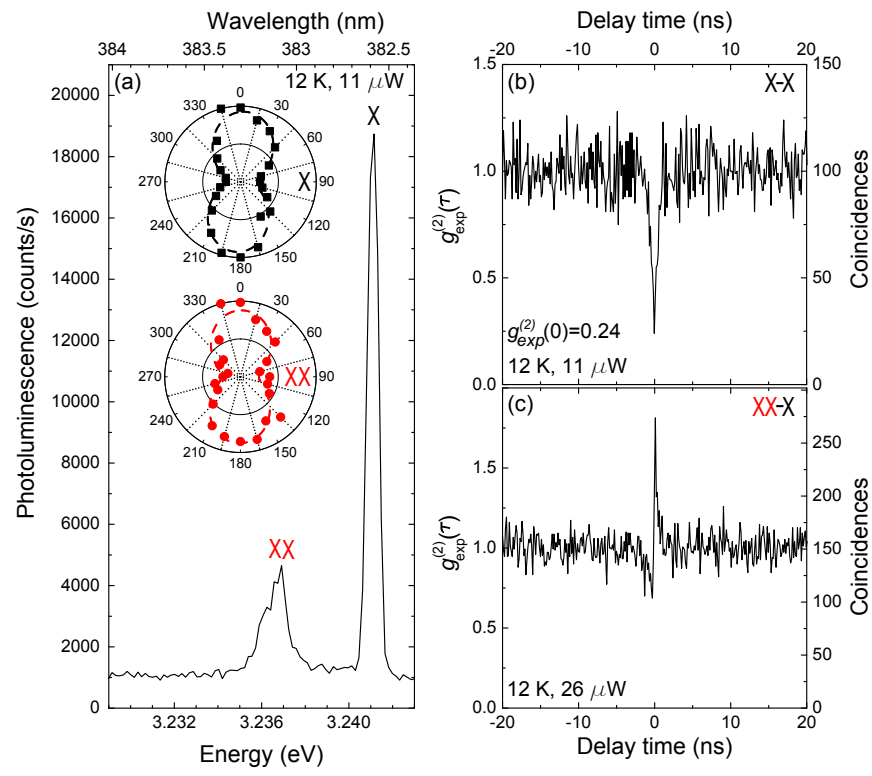

FIG. 2. (color online) a) $\mu \mathrm{PL}$ spectrum recorded with a PMT and a photon counter $(\rho=0.94$ for X). b) Autocorrelation histogram of X with $g_{\text {exp }}^{(2)}(0)=0.24$. b) Cross-correlation histogram of XX and X. The two inserts show the polarization resolved data for X (black squares) corresponding to $84 \%$ degree of polarization and XX (red circles) corresponding to $85 \%$ degree of polarization. The fitted curves are of the form $I(\theta)=I_{\text {min }} \cdot \sin ^{2}(\theta-\varphi)+I_{\max } \cdot \cos ^{2}(\theta-\varphi)$, where $I_{\text {min }}^{X}=0.20$, $I_{\max }^{X}=0.93, \varphi^{X}=3.9^{\circ}, I_{\min }^{X X}=0.25, I_{\max }^{X X}=0.88$ and $\varphi^{X X}=1.2^{\circ}$. The concentric circles have the radii of 0.5 .

Here, square brackets are used to distinguish the value $g_{m}^{(2)}[0]$ obtained by integration over a finite bin width from the corresponding value $g_{m}^{(2)}(0)$ for an infinitesimal bin width.

The exciton lifetime $\left(\tau_{x}\right)$ ultimately sets the upper frequency of the single photon emitter. It is clear from 2 that $\tau_{x}$ can be determined as the extrapolated value of $\tau_{c}$ at zero laser excitation power, corresponding to vanishing pump rate $W$ [17]. For this purpose, TCSPS auto correlation histograms of $\mathrm{X}$ were measured for different excitation powers and $\tau_{c}$ was extracted by fitting the data with 3 using $\tau_{c}$ as the only fitting parameter. The other parameters involved in 3, i.e. the instrument time constant $\tau_{i}$ and the relative exciton signal $\rho$, are known from independent measurements or instrument settings. Figure 4 shows the linear dependence of the anti-bunching rate $\left(1 / \tau_{c}\right)$ with increasing laser power, corresponding to an exciton life time of $\tau_{x}=0.7$ ns.

A high relative signal $(\rho \approx 1)$ is essential for high quality single photon emission, and our results show that $\rho$ is reduced from 0.92 to 0.83 , when the temperature is elevated from $12 \mathrm{~K}$ to $80 \mathrm{~K}$. However, as already discussed, the value of $g_{m}^{(2)}[0]$ is also affected by the TCSPS instrument time constant $\tau_{i}$ and the histogram bin width $\tau_{B W}$ in ratio with the characteristic anti-bunching time $\tau_{c}[10]$. The non-vanishing values obtained for $g_{e x p}^{(2)}(0)$ are 

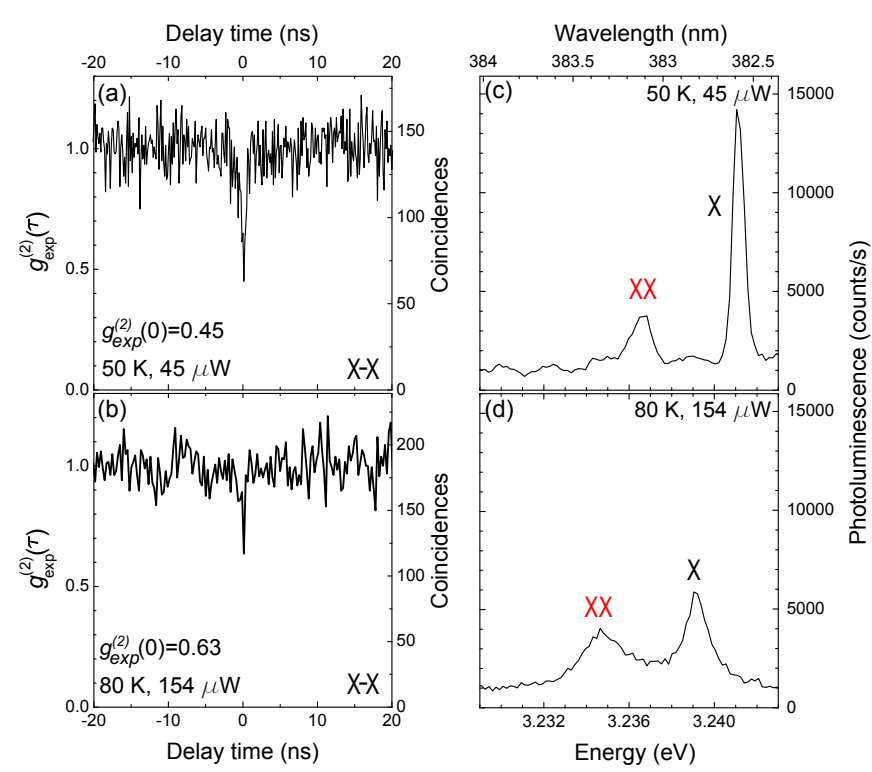

FIG. 3. (color online) a and b) Autocorrelation histograms of $\mathrm{X}$ at $50 \mathrm{~K}$ and $80 \mathrm{~K}$, respectively. c and d) $\mu \mathrm{PL}$ spectra recorded by a PMT and a photon counter at $50 \mathrm{~K}$ and $80 \mathrm{~K}$, respectively.

indeed fully explained by 4 when including the contributions from the non-zero background emission $(\rho)$ and the limited instrumental time resolution $\left(\tau_{i}\right.$ and $\left.\tau_{B W}\right)$. This implies that the investigated QD itself behaves as an essentially ideal single photon emitter up to $80 \mathrm{~K}$, but the influence from the above mentioned parameters radically increases the value of $g_{m}^{(2)}[0]$ at elevated temperatures.

The main causes for the remaining finite value of $g_{m}^{(2)}[0]$ will now be discussed for the exciton autocorrelation measurements at different temperatures (figures 2a, 3a and $3 \mathrm{~b}$ ). At $12 \mathrm{~K}$ (figure 2a), a curve fit of $g_{m}^{(2)}(\tau)$ to the experimental data yields $\tau_{c}=0.45 \mathrm{~ns}$, with a corresponding value of $g_{m}^{(2)}[0]=0.31$. In this case, the dominating factor for non-zero $g_{m}^{(2)}[0]$ is the finite instrument time constant. A deconvolution of $g_{m}^{(2)}(\tau)$, corresponding to $\tau_{i}=0$, yields the significantly lower value $g_{m}^{(2)}[0]=0.17$, but a reduction of the background emission $(\rho=1)$ or reduction of the bin width $\left(\tau_{B W}=0\right)$ alone has negligible effect. Thus, faster detectors are needed to further improve the $g_{m}^{(2)}[0]$ value measured at $12 \mathrm{~K}$.

The corresponding analysis of the data at elevated temperatures yields $\tau_{c}=0.27 \mathrm{~ns}$ and $g_{m}^{(2)}[0]=0.46$ for $50 \mathrm{~K}$ (figure 3a) and $\tau_{c}=0.12 \mathrm{~ns}$ and $g_{m}^{(2)}[0]=0.69$ for 80 K. For these temperatures, both the uncorrelated background and the instrumental time constant contribute almost equally to the finite value of $g_{m}^{(2)}[0]$. It should be noted that the influence from the instrumental time constant as well as the bin width becomes successively more significant at higher temperatures when $\tau_{c}$ becomes small.

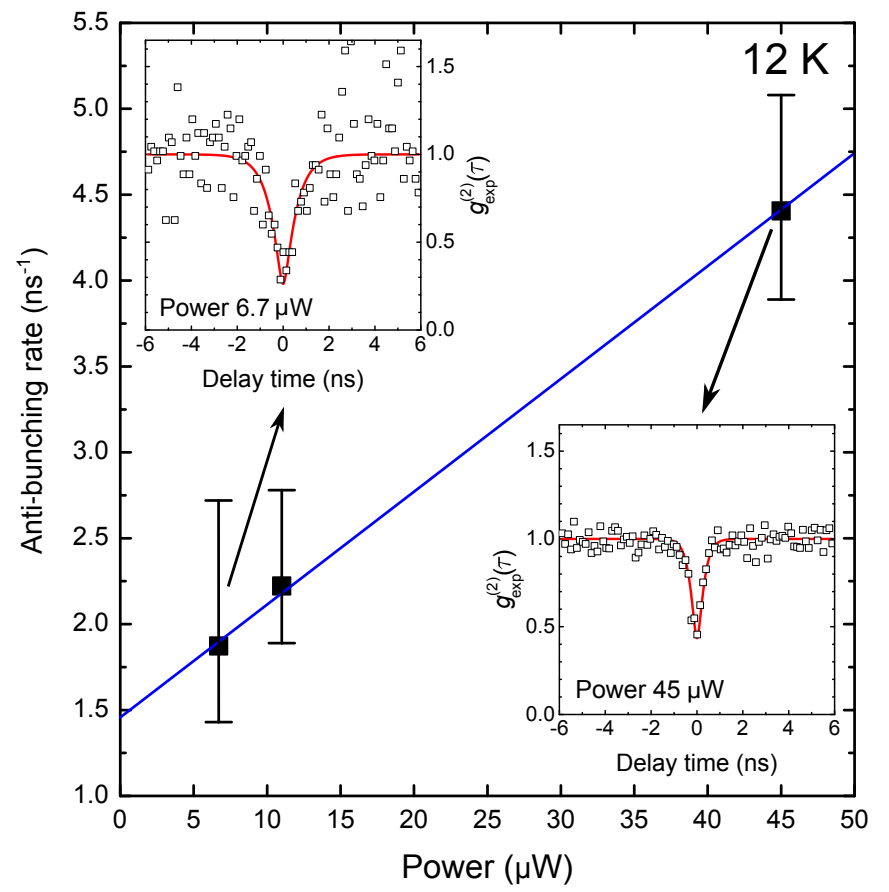

FIG. 4. (color online) The anti-bunching rate $\left(1 / \tau_{c}\right)$ extracted from the TCSPS histograms and plotted against the excitation laser power. The error bars define $95 \%$ confidence intervals for $1 / \tau_{c}$ of the best curve fit. The exciton lifetime determined as the extrapolated anti-bunching time at zero power is $\tau_{x}=0.7_{-0.28}^{+0.55} \mathrm{~ns}$, where the error refers to the maximum and minimum gradients of a line within the error bars. The histograms for the first and last data points are shown as inserts, with the fitted curve $g_{m}^{(2)}(\tau)$ shown as a red solid line. The characteristic anti-bunching times with $95 \%$ confidence intervals are $\tau_{c}=0.53 \pm 0.17 \mathrm{~ns}, \tau_{c}=0.45 \pm 0.09 \mathrm{~ns}$, and $\tau_{c}=$ $0.23 \pm 0.03 \mathrm{~ns}$, for the successively increased powers.

\section{CONCLUSIONS}

In summary, we have presented an approach to screen the background emission from GaN micropyramids by a metal film which dramatically improved the single photon characteristics ( to $g_{e x p}^{(2)}(0)=0.24$ ) of the linearly polarized excitonic emission from an InGaN QD located at the apex of a pyramid. Furthermore, we provide the first evidence of a biexciton in this QD system (with $4.5 \mathrm{meV}$ binding energy), as characterized by a typical bunching pattern in the cross correlation between the exciton and biexciton emission lines. Single photon characteristics remain up to $50 \mathrm{~K}$, but the measured second order coherence function at zero delay time significantly increases at elevated temperature, mainly due to a reduced peak intensity of the QD emission that lowers the signal-tobackground ratio and the reduced anti-bunching time that makes the limited time resolution more significant. 


\section{ACKNOWLEDGMENTS}

The authors H.M., K.F.K., and P.O.H. acknowledge financial support from the Carl Trygger Foundation for Scientific Research, the Swedish Research Council (VR), the Nano-N consortium funded by the Swedish Foundation for Strategic Research (SSF), and the Knut and
Alice Wallenberg Foundation. We acknowledge support from the Swedish Government Strategic Research Area in Materials Science on Functional Materials at Linkping University (Faculty Grant SFO-Mat-LiU \# 2009-00971). T.J. gratefully acknowledges financial support from the Font-D at Linköping University.
[1] Eisaman M D, Fan J, Migdall A and Polyakov S V 2011 Review of Scientific Instruments 82071101 ISSN 00346748 URL https://lt.ltag.bibl.liu.se/login? url=http://search.ebscohost. com/login.aspx? direct $=$ true\&db=aph\&AN $=63502292 \&$ site $=$ ehost - live

[2] Gisin N, Ribordy G, Tittel W and Zbinden H 2002 Rev. Mod. Phys. 74(1) 145-195 URL http://link.aps.org/ doi/10.1103/RevModPhys.74.145

[3] Amloy S, Karlsson K F and Holtz P O 2013 ArXiv e-prints (Preprint 1311.5731) URL http://adsabs. harvard.edu/abs/2013arXiv1311.5731A

[4] Holmes M J, Choi K, Kako S, Arita M and Arakawa Y 2014 Nano Letters 14 982-986 (Preprint http://pubs.acs.org/doi/pdf/10.1021/nl404400d) URL http://pubs.acs.org/doi/abs/10.1021/nl404400d

[5] Deshpande S, Das A and Bhattacharya P 2013 Applied Physics Letters 102161114 URL http://scitation.aip.org/content/aip/journal/ apl/102/16/10.1063/1.4803441

[6] Deshpande S and Bhattacharya P 2013 Applied Physics Letters 103241117 URL http://scitation.aip.org/ content/aip/journal/apl/103/24/10.1063/1.4848195

[7] Lundskog A, Palisaitis J, Hsu C W, Eriksson M, Karlsson K F, Hultman L, Persson P O A, Forsberg U, Holtz P O and Janzen E 2012 NANOTECHNOLOGY 23305708 ISSN 0957-4484

[8] Hsu C W, Lundskog A, Karlsson K F, Forsberg U, Janzen E and Holtz P O 2011 Nano Letters $11 \quad 2415-2418 \quad$ (Preprint http://pubs.acs.org/doi/pdf/10.1021/nl200810v) URL http://pubs . acs . org/doi/abs/10.1021/nl200810v

[9] Lundskog A, Hsu C W, Fredrik Karlsson K, Supaluck A, Nilsson D, Forsberg U, Olof Holtz P and
Janzn E 2014 Light: Science and Applications $\mathbf{3}$ 139 URL http://www . nature.com/lsa/journal/v3/n1/ full/lsa201420a.html

[10] Jemsson T, Machhadani H, Karlsson K F, Hsu C W and Holtz P O 2014 Applied Physics Letters 105 081901 URL http://scitation.aip.org/content/aip/ journal/apl/105/8/10.1063/1.4893476

[11] Renker D and Lorenz E 2009 Journal of Instrumentation 4 P04004 URL http://stacks.iop.org/1748-0221/4/ $i=04 / a=P 04004$

[12] Hanbury Brown R and Twiss R Q 1956 Nature 177 2729 URL http://www.nature.com.lt.ltag.bibl.liu. se/nature/journal/v177/n4497/pdf/177027a0.pdf

[13] Hsu C W, Moskalenko E S, Eriksson M O, Lundskog A, Karlsson K F, Forsberg U, Janzen E and Holtz P O 2013 APPLIED PHYSICS LETTERS 103013109 ISSN 0003-6951

[14] Amloy S, Karlsson K F, Andersson T G and Holtz P O 2012 Applied Physics Letters 100021901 URL http://scitation.aip.org/content/aip/journal/ apl/100/2/10.1063/1.3675572

[15] Brouri R, Beveratos A, Poizat J P and Grangier P 2000 Opt. Lett. 25 1294-1296 URL http://ol.osa.org/ abstract. cfm?URI=ol-25-17-1294

[16] Michler P, Imamoglu A, Mason M, Carson P, Strouse G and Buratto S 2000 NATURE 406 968-970 ISSN 00280836

[17] Lounis B, Bechtel H, Gerion D, Alivisatos P and Moerner W 2000 Chemical Physics Letters 329399 - 404 ISSN 0009-2614 URL http://www.sciencedirect.com/ science/article/pii/S0009261400010423 